\title{
Ergebnisse paläobotanischer Untersuchungen zur Stratigraphie und Ökologie des Würms im Oberrheingraben zwischen Karlsruhe und Mannheim
}

\author{
Meinrad Küttel, Manfred Löscher \& Adam Hölzer *) \\ Würmian, peat, gyttja, sand, pollen diagrams, paleoenvironment, peat bogs, lakes, forests, paleoclimate \\ Upper Rhine Valley, Baden-Wurttemberg \\ TK 25: Nr. 6617, 6716, 6717, 6816, 6916
}

Kurzfassung: Mit paläobotanischen Untersuchungen (Pollen- und Großrestanalysen) am Profil Rösbach, nw Leimen, konnte ein Mittelwürm-Stadial überleitend zu einem föhrenreichen Mittelwürm-Interstadial nachgewiesen werden. Das Interstadial ist am ehesten mit Hengelo zu korrelieren. Die gefundene Verlandungsfolge zeigt einen Wechsel von einem kalkreichen stehenden Gewässer zu einem basenreichen Niedermoor.

In verschiedenen Einzelproben aus 10 bis $34 \mathrm{~m}$ Tiefe unter dem Grundwasserspiegel wurden Waldpollenspektren mit hohem Koniferenanteil und geringerem Anteil an Pollen mesophiler Laubhölzer gefunden. Die zeitliche Stellung dieser Einzelproben ist nicht klar. Im Vergleich zur Grande Pile kommen Ende Eem, St. Germain I und St. Germain II in Frage.

[Results of Paleobotanical Investigations on Würmian Stratigraphy and Ecology in the Upper Rhine Valley between Karlsruhe and Mannheim]

Abstract: Paleobotanical investigations (pollen- and macrofossilanalysis) of the Rösbach profile, nw Leimen, show a sequence of Middle Würmian stadial leading to Middle Würmian interstadial rich in pine. The interstadial can at best be correlated with the Hengelo-Interstadial. Sediments have been found, showing transition of a hard water pond to a fen rich in bases. Pollenanalysis of several single samples from 10 to $34 \mathrm{~m}$ below the ground water level, yieldied pollen spectra of forests with high amounts of conifers and lower amounts of mesophytic deciduous trees. Concerning the age of this samples, in comparing with the diagram of the Grande Pile, it can be thought of the end of the Eemian, of St. Germain I or of St. Germain II.

*) Anschriften der Autoren: Dr. M. KÜTTEL, Jägerweg 16, D - 3113 Suderburg. - Dr. M. LÖSCHER, Max-RegerWeg 3, D - 6906 Leimen-St. Ilgen. - Dr. A. HöLZER, Landessammlungen für Naturkunde, Erbprinzenstraße 13, D - 7500 Karlsruhe.

\section{Forschungsstand}

Die quartären Sedimente des nördlichen Oberrheingrabens (Abb. 1) sind durch zahlreiche Bohrungen (Erdöl, Grundwasser, Kies) in ihrem lithologisch-stratigraphischen Aufbau schon seit längerem recht gut bekannt (z. B. BARTZ 1959; SCHNEIDER \& SCHNEIDER 1975; ARMBruster et al. 1977 und andere).

In den letzten Jahren wurden zur stratigraphischen Gliederung des relativ leicht zugänglichen Jungpleistozäns des nördlichen Oberrheingrabens hauptsächlich Holzreste, Schneckenschalen und Säugerknochen aus den zahlreichen Kiesgruben, bzw. tieferen Baugruben ausgewertet (LÖSCHER et al. 1980; LÖSCHER, 1981; v. KÖNIGSWALD et al. 1982).

Relativ früh sind auch schon pollenführende Ablagerungen in diesem Gebiet untersucht worden (STARK 1926; Firbas 1934; OBERDORFER 1934; ROTHSCHILD 1936; BAAS 1938), wobei jedoch das Probenmaterial aus Deckschichten auf der eiszeitlichen Niederterrasse bzw. aus der holozänen Rheinaue stammt, also höchstens spätglaziales bzw. holozänes Alter aufweist. Gleiches gilt auch für die Profile von LESSMANN (1983).

Pollenführende Sedimente aus tieferen Lagen und damit älteren Zeitabschnitten (VON DER BRELIE in BARTZ 1976; BARTZ 1982; ferner einzelne Beiträge von SCHEDLER in LÖSCHER et al. 1980, und von SCHLOSS in LÖSCHER et al. 1983) wurden bis jetzt noch nicht so oft untersucht, was sicher damit zusammenhängt, daß die systematische Erschließung der Grundwasservorräte und der Kiesablagerungen verstärkt erst nach dem 2. Weltkrieg einsetzten, andererseits das pollenführende Probenmaterial nie im Verbund, sondern meist nur gestört anfällt. 


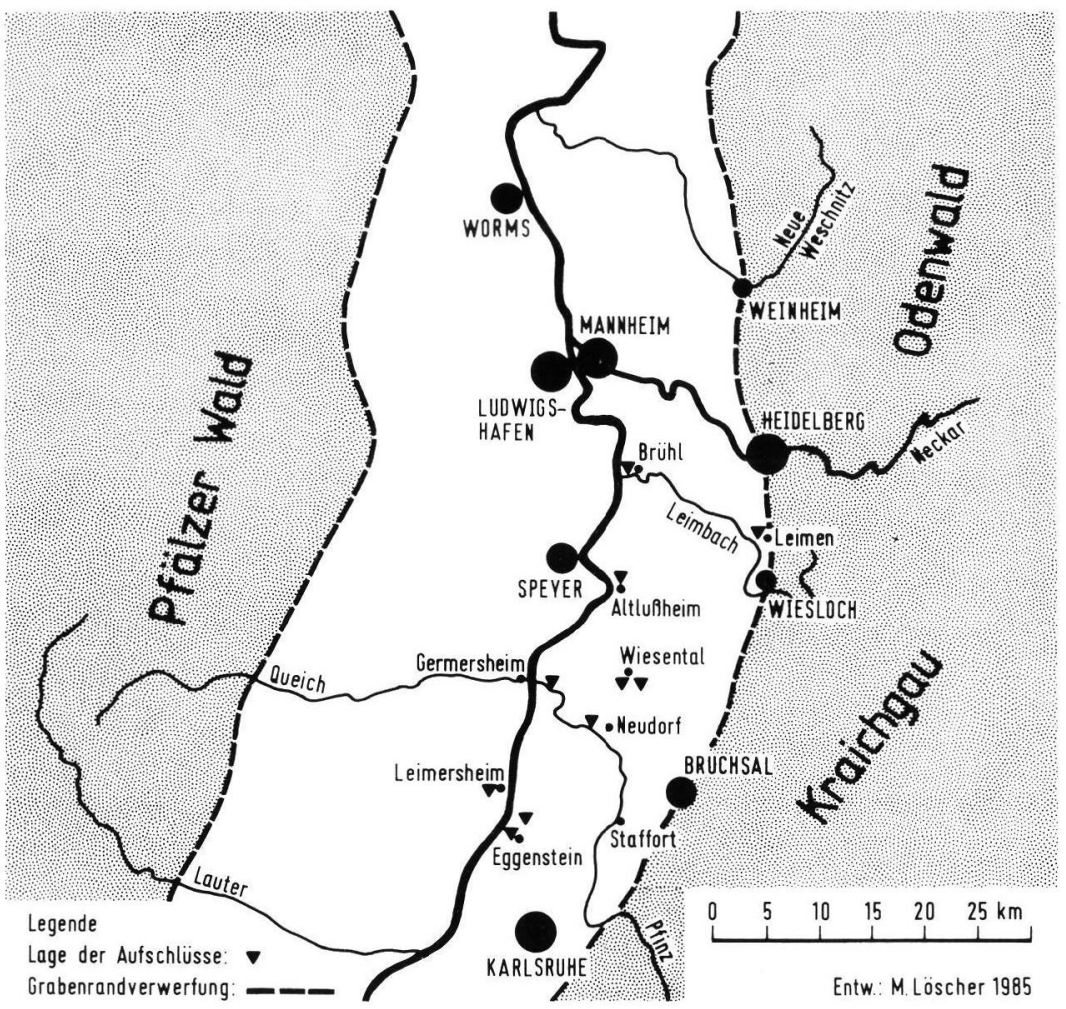

Abb. 1: Geographische Übersicht.

\section{Vegetation des Untersuchungsgebietes}

Das nördliche Oberrheingebiet ist heute weitgehend Kulturlandschaft. Nach einer Karte der potentiellen natürlichen Vegetation (MÜLLER et al. 1974) würden auf dem Neckarschwemmkegel Eichen-Hainbuchenmischwälder wachsen, sodann auf der Dünenlandschaft südlich von Mannheim ein Buchen-Eichenwald im Kontakt mit Wintergrün-Kiefernwald und entlang des Rheines Eichen-Ulmen- und Silberweiden-Auewald.

\section{Stratigraphie}

Im Bereich des Neckarschwemmfächers wurde das Jungpleistozän bereits stratigraphisch untergliedert (LÖSCHER et al. 1980; LÖSCHER 1981). Nach jetzigem Stand gilt diese Gliederung, stellvertretend dafür die Kiesgrube Dr. Bauer, 1,5 km südöstlich Wiesenthal (Abb. 2), im Prinzip für den gesamten rechtsrheinischen Teil zwischen Karlsruhe und Darmstadt, allerdings unter Berücksichtigung folgender Tatsachen:

a) Die jungpleistozänen Schichten fallen von W nach E ein, weil der nördliche Rheingraben am Ostrand stärker absinkt. Linksrheinisch sind die jungquartären Schichten des Rheins stellenweise nur ca. $10 \mathrm{~m}$ mächtig, während sie am östlichen Grabenrand stellenweise mindestens $40 \mathrm{~m}$ mächtig werden.

b) Im Bereich der Rheinaue wurden die würmeiszeitlichen Kiese - zumindest stellenweise - bis auf mindestens 10 bis $12 \mathrm{~m}$ Tiefe unter dem Grundwasserspiegel ausgeräumt und durch holozäne Ablagerungen ersetzt.

c) In der Nähe des Ostrandes sind stellenweise tektonisch nicht sehr tief abgesunkene Randschollen nur wenige Meter von quartärem Schotter bedeckt.

Da das Probenmaterial vorwiegend aus dem Bereich unterhalb des Grundwasserspiegels stammt, zeigen die Profile teilweise skizzenhaften Charakter, der allerdings durch ausgiebige Befragung des Kiesgrubenpersonals abgesichert wurde.

Auffallend ist, daß in allen Kiesgruben mit entsprechender Abbautiefe ein Horizont mit zahlreichen Eichenstämmen vorkommt, die folgende Eigenschaften aufweisen: 


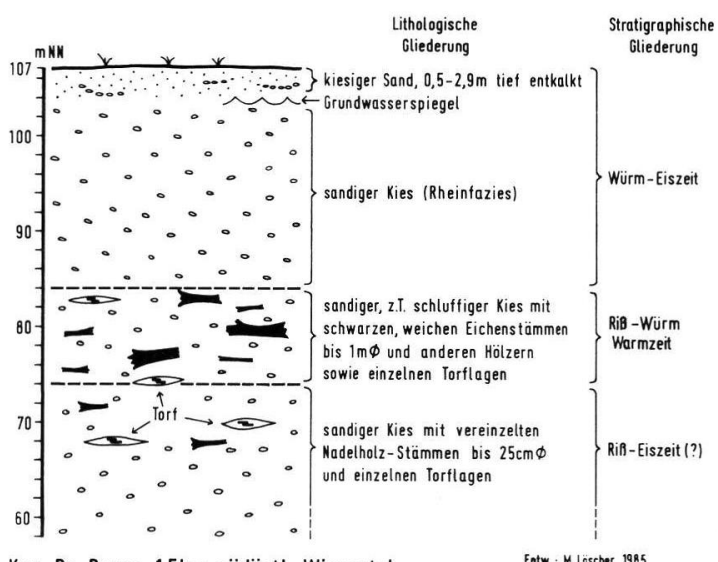

Kgr. Or. Baver, 1,5km südöstl. Wiesental

Abb. 2: Die Schichtenfolge in der Kiesgrube Dr. Bauer, se Wiesenthal.

a) dunkelbraune bis schwarze Farbe

b) sehr weich (sowohl in feuchtem als auch in getrocknetem Zustand)

c) Pyritnadeln parallel zu den Leitungsbahnen.

Diese eichenführende Schicht enthält außerdem warmzeitliche Säugerreste und warmzeitliche Konchylien. Sie hat für die stratigraphische Gliederung des Jungquartärs die Funktion eines Leithorizontes. Da sie die erste, eindeutig warmzeitliche Schicht (von oben her betrachtet) ist, wurde sie von LÖSCHER (1981) ins Riss/Würm-Interglazial gestellt. Lithologich gesehen liegt diese Schicht im unteren Teil des vorwiegend sandig-kiesigen sogenannten „oberen Grundwasserleiters" und direkt auf dem vorwiegend schluffigfeinsandig sog. „oberen Ton”, soweit dieser überhaupt vorhanden ist.

In diesem Zusammenhang sei daran erinnert, daß BARTZ (1959) und andere bisher den gesamten „oberen Grundwasserleiter” in die Würmkaltzeit und den „oberen Ton” in das Riss/Würm-Interglazial gestellt haben.

Während über die Fauna und Flora des Riss/WürmInterglazials im nördlichen Oberrheingraben - trotz der nicht gerade idealen Abbauverhältnisse unter dem Grundwasserspiegel - einige konkrete Aussagen möglich sind, ist das Altwürm bis jetzt nicht gut faßbar.

Dafür konnten die Fauna und vor allem die Flora des Mittelwürms durch zahlreiche ${ }^{14} \mathrm{C}$-datierte Holzreste wieder recht gut rekonstruiert werden (LÖSCHER et al. 1983), weil diese Schichten vor allem im Neckar- schwemmfächer über oder nur knapp unter dem Grundwasserspiegel liegen. In der Kiesgrube Heckmann, nordwestlich Mannheim-Wallstadt, ergab sich nach Makroresten folgende Gliederung:

a) vor ca. 54000-ca. 42500 J. v. h. ein Nadelmischwald ( $44 \%$ Kiefern, $41 \%$ Fichte und $12 \%$ Tanne und ca. 4,5\% Laubbäume mit einigen thermophilen Elementen).

b) von ca. 42500 - ca. $27000 \mathrm{~J}$. v. h. ein fast reiner Kiefernwald.

c) für die Zeit von ca. 27000-ca. $11000 \mathrm{~J}$. v. h. liegen bis jetzt aus dem nördlichen Oberrheingraben keine Holzreste vor. Dies dürfte für einige Abschnitte des Spätglazials (speziell Bölling und Alleröd) auf Fundlücken zurückzuführen sein. $\mathrm{Zu}$ beachten ist, daß die Altersangaben auf ${ }^{14} \mathrm{C}$-Datierungen beruhen, die im Altersbereich vor etwa 40000 Jahren mit einer gewissen Unsicherheit behaftet sind.

\section{Neue Untersuchungen}

\subsection{Herkunft der Proben}

Die Einzelproben (Aufschlüsselung der Lokalitätennummer im Diagramm, Abb. 7, s. Tabelle 1) stammen aus dem Liegendteil des „oberen Grundwasserleiters". Wegen des Nassbaggerei-Verfahrens ist es im Einzelfall nicht möglich, mit genügender Sicherheit festzustellen, ob diese Proben aus dem eichenholzführenden Horizont stammen oder direkt darüber. Das bedeutet aber auch, daß die stratigraphische $\mathrm{Zu}$ ordnung dieser Einzelproben aus lithologischer Sicht nicht genügend eindeutig ist. Nach der Schichtung wurde die vertikale Achse festgestellt, so daß an größeren Blöcken kleine Profile entnommen werden konnten. Die Profilstücke sind im Diagramm (Abb. 8) mit durchgehender Lithologie-Säule dargestellt (z. B. Proben $2 / 0$ bis $2 / 15$ ), wobei allerdings unbekannt ist, ob sie stratigraphisch korrekt aufgezeichnet sind. Beispielsweise könnte das kleine Profil der Lokalität 2 in Wirklichkeit ebensogut auf den Kopf stehen. Dies engt die Deutung der Einzelproben natürlich wesentlich ein, zumal auch die stratigraphischen Beziehungen der einzelnen Lokalitäten zueinander unbekannt sind. Das Profil Rösbach, nw Leimen (s. Abb. 1), wurde im Graben als Säule an einer Stichwand entnommen (s. Abb. 3). So konnte die Profilsäule im Labor parallel für Pollenanalyse und Glühverlust beprobt werden. Das restliche Material wurde für die Großrestanalyse und die geochemischen Untersuchungen verwendet. 


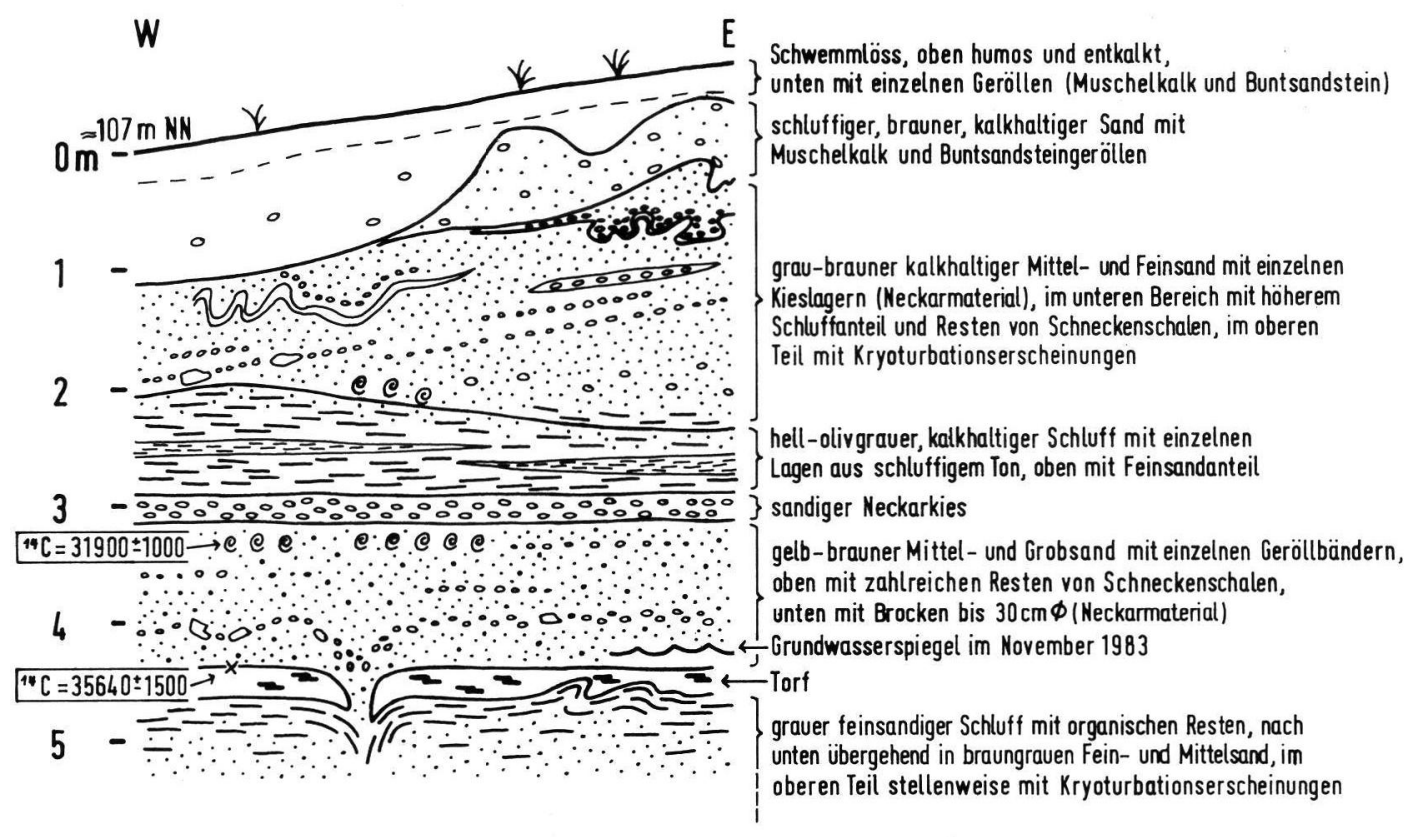

\section{Kanalisationsgraben im Gewann Rösbach, NW Leimen}

Entw.: M. Löscher 1985

Abb. 3: Lithologische Verhältnisse im Aufschluß Kanalisationsgraben Rösbach.

Das Profil Rösbach (RÖS) stammt aus dem Torfband und den unterlagernden Schichten in ca. $4.5 \mathrm{~m}$ Tiefe.

\subsection{Methodische $\mathrm{H}$ inwe is e}

Die Proben für die Pollenanalyse wurden nach der in Hohenheim gängigen Methodik aufbereitet (vgl. FRENZEL 1964, neuere Darstellung in KÜTTEL 1983). Die Proben für den Glühverlust wurden 24 h bei 104 Grad getrocknet und $4 \mathrm{~h}$ bei $550 \mathrm{Grad}$ verascht. Die Ergebnisse sind in den Diagrammen (Abb. 4 und Abb. 7) dargestellt. Die Prozentberechnung der pollenanalytischen Daten wurde so gewählt, daß stets die Typen, deren Anteile berechnet werden sollten, in die entsprechende Grundsumme eingeschlossen wurden. Im speziellen Fall heißt hier das, daß Baumpollen (mit Strauchpollen) und Nichtbaumpollen auf der Summe des Baum- und des Nichtbaumpollens berechnet wurde. Die Algen Pediastrum und Botryococcus wurden auf die Summe des Pollens und der Sporen plus die der Algen bezogen. Die Vorteile dieser Berechnungsweise liegen auf der Hand und sind längst diskutiert worden (z. B. KÜTTEL 1983). Die Ergebnisse des Profils Rösbach sind in herkömmlicher Weise dargestellt. Außer im Hauptdiagramm, das vor allem das Verhältnis zwischen dem Baumpollen und dem Nichtbaumpollen zeigen soll, beträgt eine Einheit $1 \%$. Im Hauptdiagramm sind es $10 \%$.
Für die Abbildung der Einzelproben oder der kurzen Profilstücke wurde die Säulendarstellung gewählt. Hier beträgt die Einheit bei den weißen Säulen $10 \%$. Die schwarzen Säulen sind 10-fach überhöht. Die Anordnung der Typen basiert auf ökologischen Gruppen.

Das Pollendiagramm Rösbach wurde in Pollenzonen gegliedert, die entsprechend den stratigraphischen Prinzipien als Biozonen $\mathrm{zu}$ verstehen sind. Die chronostratigraphische Einstufung fußt auf Kriterien, die weiter hinten diskutiert werden sollen.

Zur Berechnung der Diversität und der Evenness (Abb. 5), sowie des theoretischen Ansatzes dazu sei auf KÜTTEL (1984b) verwiesen. Für die Großrestanalysen (Abb. 6) wurde das Profil in Scheiben von $1 \mathrm{~cm}$ Dicke aufgeschnitten. Etwa $25 \mathrm{ccm}$ wurden mit 5 \% iger Kalilauge leicht erwärmt. Das Material wurde danach nicht durch Sieben, wie in früheren Arbeiten (HÖLZER \& SCHLOSS 1981), sondern durch mehrmaliges Dekantieren nach Zugabe von destilliertem Wasser wieder von der $\mathrm{KOH}$ gereinigt. Die Proben wurden unter einem Stereomikroskop verlesen. Als Bestimmungshilfen neben der Vergleichssammlung dienten: AALTO (1970); BERGGREN (1969); BERTSCH 


\section{RÖSBACH}

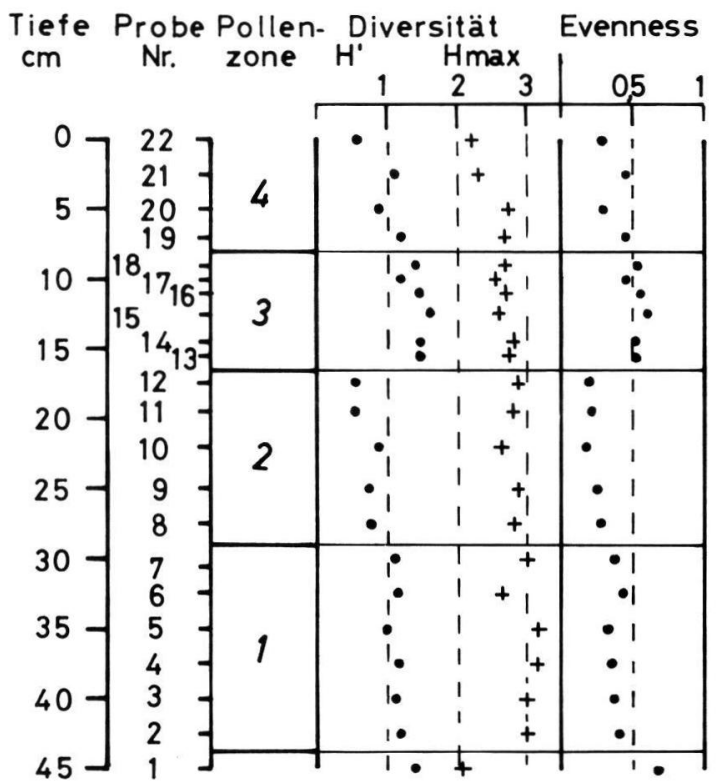

Abb. 5: Veränderung von Diversität und Evenness des Pollendiagramms Rösbach (RÖS).

(1941); Grosse-BraUCKMANN (1972); KaC et al. (1965); Nilsson u. HJELmQvist (1967); NYHOlm (1954-1969).

Der Anteil der einzelnen Arten wurde in Bezug auf die Gesamtpflanzenmasse in Prozent geschätzt. Einzelfunde wurden durch das Zeichen „, " dargestellt.

Für die chemischen Analysen wurde ein Teil der $1 \mathrm{~cm}$ Scheiben zuerst bei 95 Grad Celsius getrocknet, dann in einer Achatmühle gemahlen. Verascht wurde bei 550 Grad während 12 Stunden. Die Silizium-Bestimmung erfolgte nach Schmelzen mit $\mathrm{NaOH}$ im Nickeltiegel photometrisch mit Ammoniummolybdat. Der Stickstoff wurde nach Aufschluß nach ALLEN (1974) photometrisch über die Indophenolblau-Methode bestimmt.

\subsection{E rge b $\mathrm{n}$ is se}

\subsubsection{Profil RÖSBACH}

(RÖS, $0 \mathrm{~cm}$ bedeutet Oberkante des Torfes)

Lithologie:

$0-10 \mathrm{~cm}$ Flachmoortorf mit von unten nach oben zunehmendem mineralischen Anteil

$10-45 \mathrm{~cm}$ siltig-sandige Gyttja mit von unten nach oben zunehmendem organischem Gehalt. Für Details wird auf die Glühverlustkurve verwiesen.

\section{Pollenzonen:}

Auf den ersten Blick läßt sich das gesamte Diagramm (Abb. 4) in zwei Zonen teilen, nämlich eine untere Zone mit Baumpollenwerten unter $30 \%$ und eine obere mit Baumpollenwerten über $40 \%$. Bei genauerer Betrachtung drängt sich aber doch eine feinere Gliederung auf, die durch den Verlauf der Diversitätskurve (Abb. 5) gestützt wird.

\section{RÖS-1 Juniperus-Artemisia-Zone}

Pollen-Proben $2-7$

Charakteristisch sind neben den namengebenden Typen vor allem noch Hippophaë, Plantago medial major-Typ, Thalictrum, Apiaceae und Chenopodiaceae. Die Probe Nr. 1 enthält eine etwas abweichende Pollenflora mit \%-Werten, die auf die sehr geringe Pollensumme zurückzuführen sind $(z$. B. basieren $3.1 \%$ Picea auf einem Korn).

Untere Grenze: Erste Probe mit genügender Pollenführung

Obere Grenze: Abnahme von Juniperus, Hippophä̈, Betula und verschiedenen NBP-Typen; Zunahme der Poaceae.

\section{RÖS-2 Poaceae-Zone}

Pollen-Proben 8-12

Charakteristisch sind die durchgehend sehr hohen Werte der Poaceae, die Zunahme von Menyanthes und das Verschwinden von Pediastrum und Botryococcus.

Obere Grenze: Anstieg von Betula und von Pinus; Rückgang der Poaceae; Zunahme der Cyperaceae.

\section{RÖS-3 Betula-Pinus-Zone}

Pollen-Proben 13-18

Pinus nimmt kontinuierlich zu, Betula ebenfalls. Allerdings geht Betula gegen das Ende der Zone wieder zurück. Auf der Gegenseite verlieren die Poaceae erheblich an Anteil. Dieser sprunghafte Rückgang ist nicht mit einer massiven Zunahme des Glühverlustes gekoppelt, sondern mit einem allmählichen Anstieg. Augenfällig verhalten sich die Cyperaceae. Sie erreichen ein Maximum.

Obere Grenze: Einsetzen der durchgehenden Kurve von Picea.

\section{RÖS-4 Pinus-Zone}

Pollen-Proben 19-22

Die letzte Zone ist durch die sehr hohen Werte von Pinus gekennzeichnet, Picea ist, zwar mit geringen Anteilen, doch durchgehend vorhanden. 
(யว) วเว!!

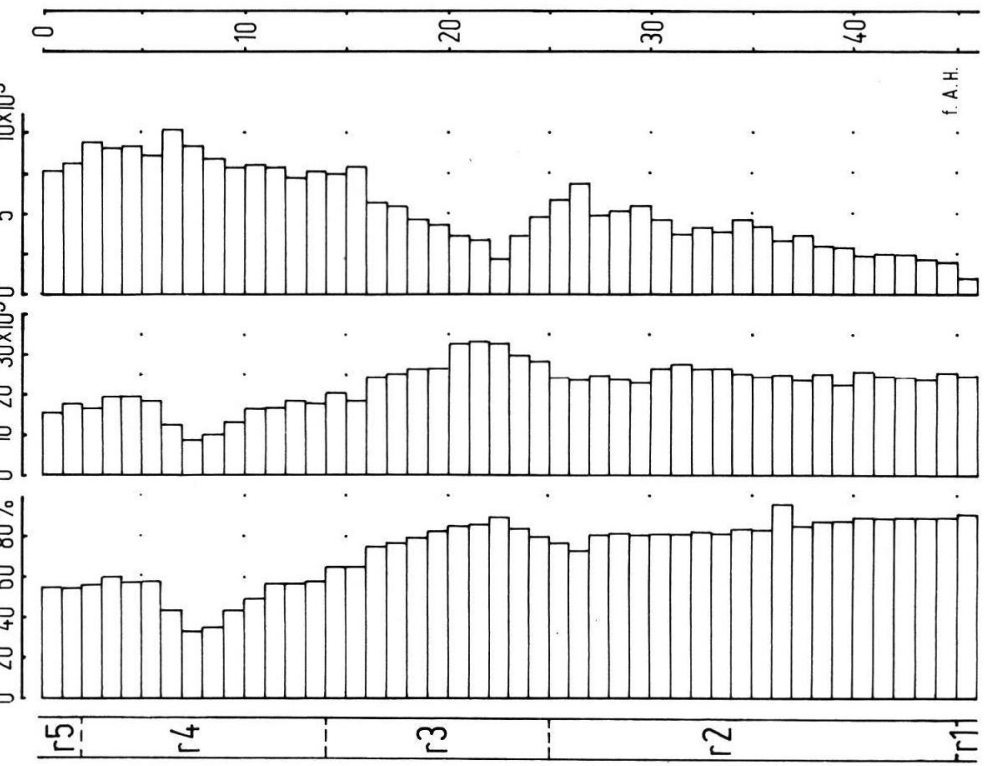

NJNOZISJySSOYg

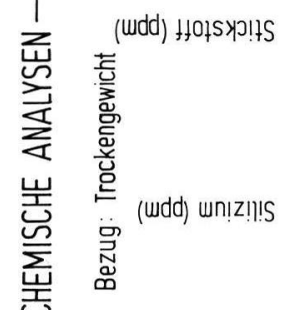

(J.05S) วपगS४

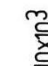

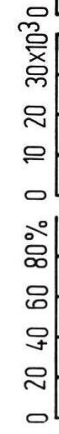

는: 혼

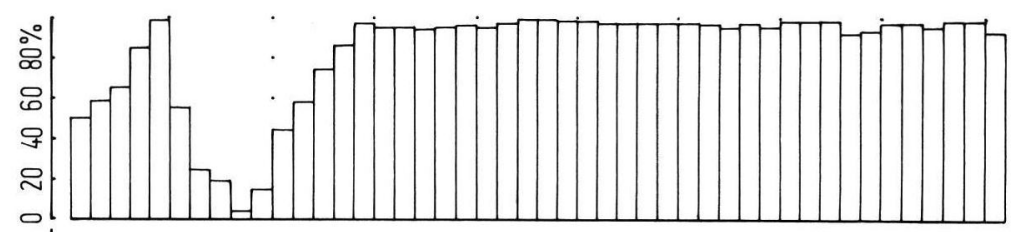

$\mathrm{Z} \mathrm{OOH}$

+ Jads unłas!nby 웅.

วъบวก-

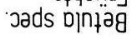

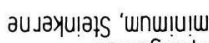
wn!uof́sods aUjayuläS 'suptDu notabowolod

uawDS 'DfD!joy!dt Saytudkuaw วิ4วกู 'DIJDJIsan XajDo $\circ$<smiles>[C]#[C+]</smiles>

บวเОБК Jads DJD4ว แnวuाயDJts vo6ja!!|ाD?

うे.
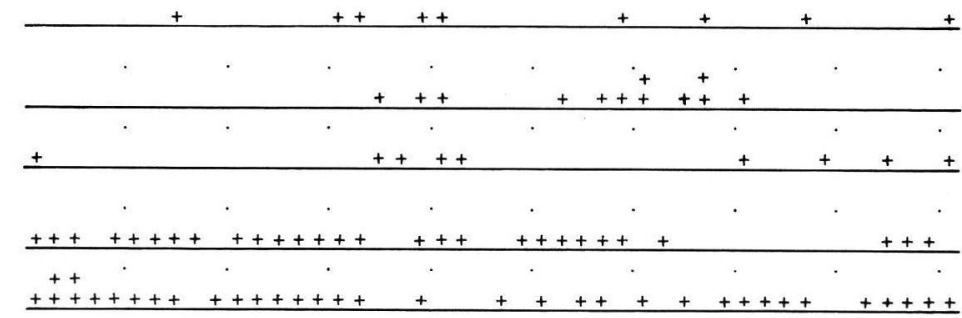

seploudjoos unipidjoos

snכunpo snpojJouddajo

SUал|0^а」 snpD|jouddaJo

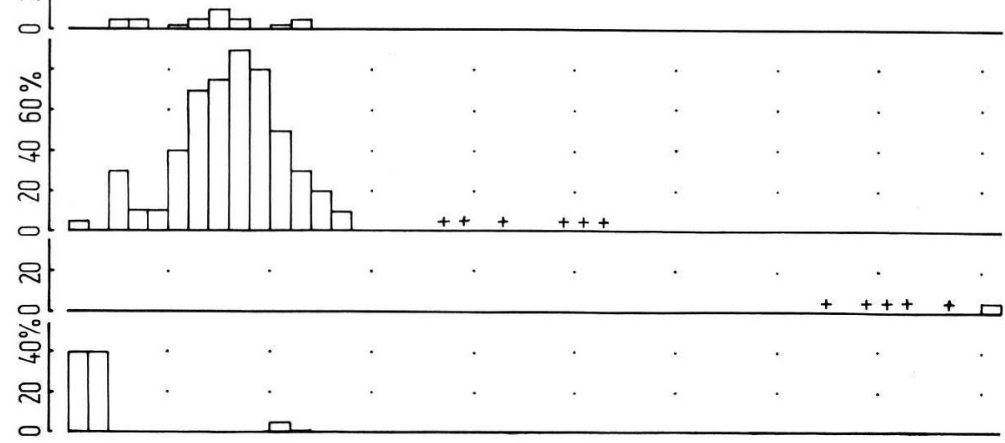


EINZELPROBEN nördl. OBERRHEINGRABEN

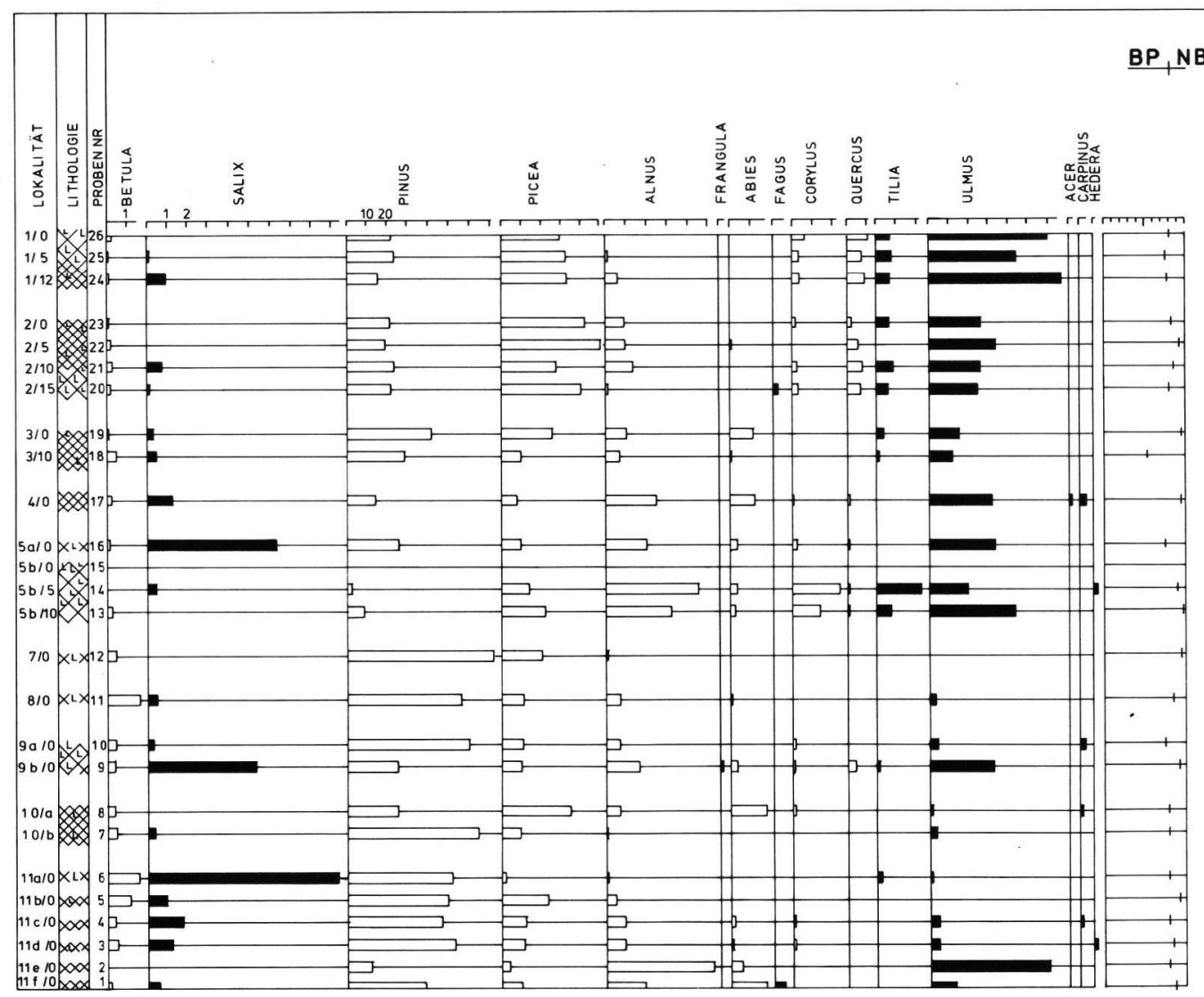

Abb. 7: Pollenspektren der Einzelproben und kurzen Profile aus dem nördlichen Oberrheingebiet.

Obere Grenze: Ende des Diagrammes.

Anmerkung: Zwischen den Zonen 3 und 4 ließe sich auch eine andere Grenzziehung begründen, nämlich zwischen den Proben 16 und 17, das heißt dort, wo Betula zurückzugehen beginnt.

\section{Makroreste:}

Das Makrorest-Diagramm Rösbach (Abb. 6) läßt sich ebenfalls in verschiedene Zonen gliedern. Sie unterscheiden sich durch unterschiedliche Dominanz der Arten.

r-1 Drepanocladus cf. aduncus-Zone

Tiefe $46 \mathrm{~cm}$

Charakteristisch ist der hohe Anteil der namengebenden Art. Dies entspricht wiederum einem besonders niedrigen Stickstoffgehalt (Abb. 6, rechts).

\section{r-2 Chara sp.-Zone}

Tiefe $26-45 \mathrm{~cm}$
Sie ist charakterisiert durch das durchgehende Vorkommen von Chara sp.

r-3 Potamogeton natans-Sparganium minimum-Zone Tiefe $15-25 \mathrm{~cm}$

Diese Zone ist mehr durch das Fehlen besonderer Arten gekennzeichnet als durch ihr Vorkommen. Es gibt aber geringe Anteile von Scorpidium scorpioides.

\section{r-4 Scorpidium scorpioides-Calliergon stramineum- Zone \\ Tiefe $3-14 \mathrm{~cm}$}

Hier dominieren die beiden namengebenden Arten. Auffallend ist auch der geringe Anteil der unbestimmbaren Arten.

\section{i-5 Drepanocladus revolvens-Zone Tiefe $1-2 \mathrm{~cm}$}

Typisch ist hier die namengebende Art und der Rückgang von Scorpidium scorpioides. 


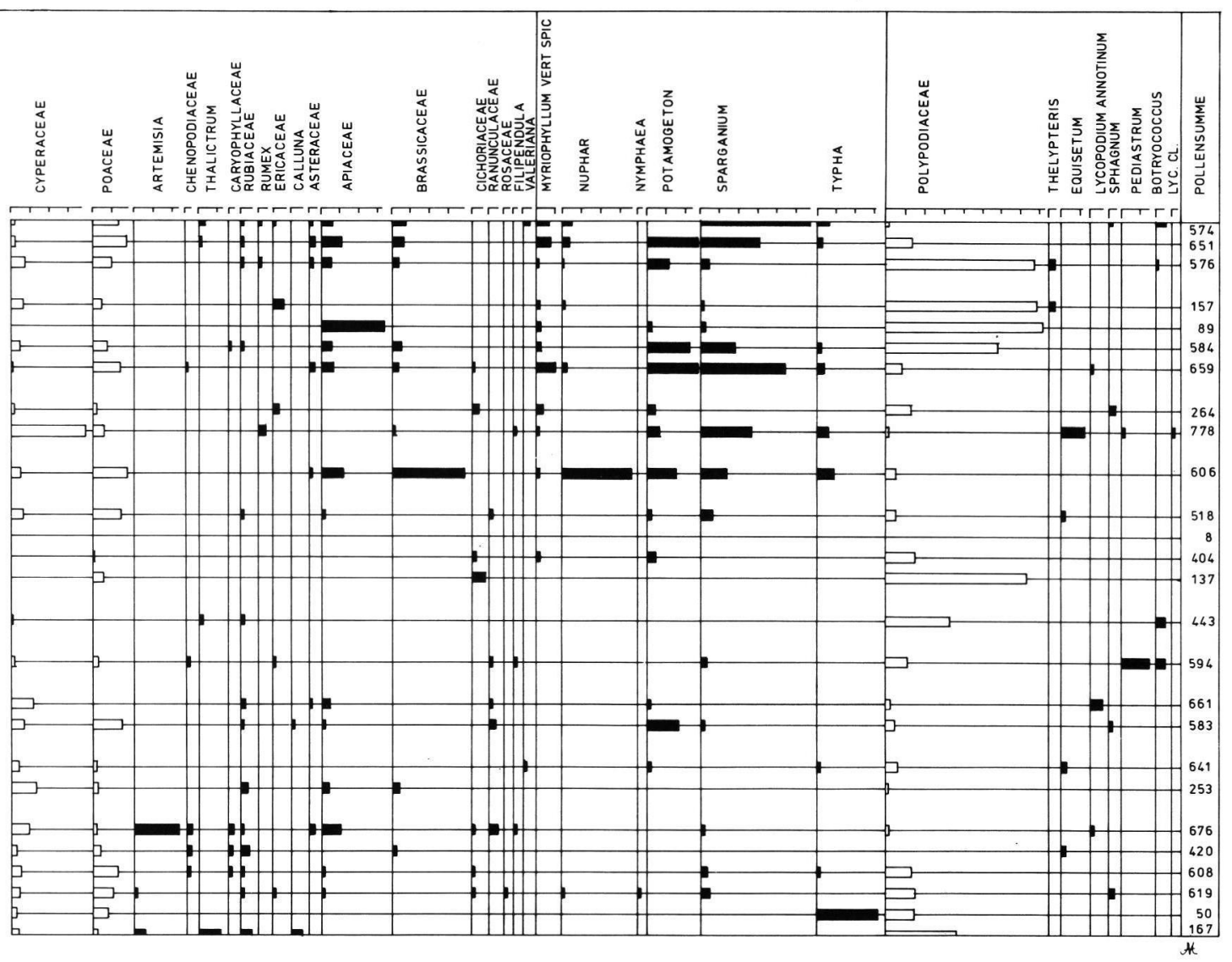

\subsubsection{Einzelproben}

Die Problematik, die mit den Einzelproben verbunden ist, wurde schon bei den Anmerkungen zur Herkunft des Materials erwähnt. Es muß aber nochmals ausdrücklich betont werden, daß die Darstellung im Diagramm (Abb. 7) kein zeitliches Aufeinanderfolgen bedeutet, außer in den Fällen der kleinen Profilsequenzen, die aber ebensogut auf dem Kopf stehen könnten. Aus diesen Gründen soll nur auf ein paar wichtige Züge eingegangen werden.

\section{Lithologie:}

Bei den meisten Proben handelt es sich um Gyttjen mit wechselndem feinklastischem Anteil. Insbesondere waren keine Flachmoortorfe dabei. Genetisch gesehen sind es Flachwassersedimente, die in Altwasserarmen gebildet wurden. Die allgemeine geomorphologische Situation spricht für diese Deutung.
Pollenanalysen:

Charakteristisch für alle Proben sind hohe Anteile der Koniferen, und zwar sowohl von Pinus als auch von Picea. Abies hingegen ist in geringerem Maße vertreten. In einzelnen Probenserien fehlt die Weißtanne fast ganz, zum Beispiel in der Kiesgrube Kühl westlich von Brühl (Proben 20-26). In den meisten Proben fanden sich auch Spuren bis ansehnliche Mengen vom Pollen thermophiler Gehölze, wobei die Hauptmenge von der Eiche stammt (bis über $10 \%$ ). Fagus wurde nur in 2 Proben gefunden. Die Summe des Baumpollens liegt um 80-90\%, ausgenommen in der Probe 18 aus der Kiesgrube Fuchs und Gross. In dieser liegen die Cyperaceae sehr hoch, was aber als durchaus lokale Erscheinung verstanden werden kann.

Innerhalb des Nichtbaumpollens scheint vor allem die reiche Wasser- und Sumpfflora erwähnenswert. Sie stützt die genetische Deutung der Sedimente. Apokrate Sippen, also Sippen, die für ihr Gedeihen in 
Tab. 1: Lokalitäten der Einzelproben nördl. Oberrheingraben (Abb. 8)

Proben-Nr.

Lokalität

Tiefe unter

Grundwasserspiegel

\begin{tabular}{|c|c|c|c|c|c|}
\hline 26 & $1 / 0$ & Kiesgrube Kühl w Brühl & & $13-14 \mathrm{~m}$ & $0 \mathrm{~cm}$ \\
\hline 25 & $1 / 5$ & Kiesgrube Kühl w Brühl & & $13-14 \mathrm{~m}$ & $5 \mathrm{~cm}$ \\
\hline 24 & $1 / 12$ & Kiesgrube Kühl w Brühl & & $13-14 \mathrm{~m}$ & $12 \mathrm{~cm}$ \\
\hline 23 & $2 / 0$ & Kiesgrube Kühl w Brühl & & $14-16 \mathrm{~m}$ & $0 \mathrm{~cm}$ \\
\hline 22 & $2 / 5$ & Kiesgrube Kühl w Brühl & & $14-16 \mathrm{~m}$ & $5 \mathrm{~cm}$ \\
\hline 21 & $2 / 10$ & Kiesgrube Kühl w Brühl & & $14-16 \mathrm{~m}$ & $10 \mathrm{~cm}$ \\
\hline 20 & $2 / 15$ & Kiesgrube Kühl w Brühl & & $14-16 \mathrm{~m}$ & $15 \mathrm{~cm}$ \\
\hline 19 & $3 / 0$ & Kiesgrube Fuchs u. Gross w Eggenstein & & $24-25 \mathrm{~m}$ & $0 \mathrm{~cm}$ \\
\hline 18 & $3 / 10$ & Kiesgrube Fuchs u. Gross w Eggenstein & & $24-25 \mathrm{~m}$ & $10 \mathrm{~cm}$ \\
\hline 17 & $4 / 0$ & Kiesgrube Erndwein nw Leopoldshofen & & $25-28 \mathrm{~m}$ & \\
\hline 16 & $5 a / 0$ & Kiesgrube Sämann s Staffort & & ca $15 \mathrm{~m}$ & \\
\hline 15 & $5 \mathrm{~b} / 0$ & Kiesgrube Sämann s Staffort & & ca $25 \mathrm{~m}$ & $0 \mathrm{~cm}$ \\
\hline 14 & $5 b / 5$ & Kiesgrube Sämann s Staffort & & ca $25 \mathrm{~m}$ & $5 \mathrm{~cm}$ \\
\hline 13 & $5 \mathrm{~b} / 10$ & Kiesgrube Sämann s Staffort & & ca $25 \mathrm{~m}$ & $10 \mathrm{~cm}$ \\
\hline 12 & $7 / 0$ & Kiesgrube Bauer se Wiesenthal & & $33-34 \mathrm{~m}$ & \\
\hline 11 & $8 / 0$ & Kiesgrube Wittmer s Wiesenthal & & $25-28 \mathrm{~m}$ & \\
\hline 10 & $9 \mathrm{a} / 0$ & Kiesgrube Pfadt w Leimersheim & & ca $12 \mathrm{~m}$ & \\
\hline 9 & $9 \mathrm{~b} / 0$ & Kiesgrube Pfadt w Leimersheim & & ca $12 \mathrm{~m}$ & \\
\hline 8 & $10 / a$ & Kiesgrube Brecht s Rheinsheim & $\mathrm{ca}$ & $10-12 \mathrm{~m}$ & \\
\hline 7 & $10 / \mathrm{b}$ & Kiesgrube Brecht s Rheinsheim & $\mathrm{ca}$ & $10-12 \mathrm{~m}$ & \\
\hline 6 & $11 \mathrm{a} / 0$ & Kiesgrube Phillip $1 \mathrm{w}$ Neudorf & & $21-22 \mathrm{~m}$ & \\
\hline 5 & $11 \mathrm{~b} / 0$ & Kiesgrube Phillip 1 nw Neudorf & ca & $20-22 \mathrm{~m}$ & \\
\hline 4 & $11 \mathrm{c} / 0$ & Kiesgrube Phillip 1 nw Neudorf & $\mathrm{ca}$ & $20-22 \mathrm{~m}$ & \\
\hline 3 & $11 \mathrm{~d} / 0$ & Kiesgrube Phillip 1 nw Neudorf & $\mathrm{ca}$ & $20-22 \mathrm{~m}$ & \\
\hline 2 & $11 \mathrm{e} / 0$ & Kiesgrube Phillip 1 nw Neudorf & $\mathrm{ca}$ & $20-22 \mathrm{~m}$ & \\
\hline 1 & $11 \mathrm{f} / 0$ & Kiesgrube Phillip 1 nw Neudorf & ca & $20-22 \mathrm{~m}$ & \\
\hline
\end{tabular}

erster Linie frei von Konkurrenz sein müssen, sind kaum von Bedeutung, was angesichts der Gehölzpollenspektren auch nicht erstaunt.

\subsection{D is kussion}

\subsection{1. a) Diagramm RÖSBACH (RÖS)}

\subsubsection{Vegetationsentwicklung}

Bei der Interpretation des Diagrammes ist grundsätzlich zwischen den ganz lokalen und den regionalen
Vegetationsveränderungen $\mathrm{zu}$ unterscheiden. Die streng lokale Vegetation ist hier die aquatische. Die Sedimentenfolge von siltiger Gyttja zu einem Flachmoortorf weist darauf hin, daß es sich um einen Teich handeln muß, der verlandet ist. Dieselbe Entwicklung ist auch im Pollendiagramm wiederzufinden.

Es beginnt mit reinen Wasserpflanzen, den Algen Pediastrum und Botryococcus, von höheren Wasserpflanzen gesellen sich dazu Potamogeton und, in niedrigem Wasser stehend, Sparganium. Auf zumindest kleine offene Wasserflächen verweist auch Utricularia. Feuchte Verhältnisse zeigt überdies Equisetum an. 
Die Zeiger offenen Wassers verschwinden mit dem stärkeren Hervortreten von Menyanthes und der Cyperaceae. Wovon diese Verlandung, die im übrigen über die Großreste ausgezeichnet nachvollzogen werden kann (siehe unten), gesteuert ist, autogen oder klimatogen, kann nicht entschieden werden. Zwar sind sowohl Menyanthes als auch Utricularia eigentliche boreale Sippen, aber die geringen Werte von Menyanthes an der Basis können auch mit fehlenden Standorten zusammenhängen. Die regionale Entwicklung der Vegetation zeigt ebenfalls eine gewisse Sukzession. Neben den hohen Werten der Poaceae und dem dominierenden übrigen Nichtbaumpollen insgesamt zeigen die Sträucher Juniperus und Hippophä eindeutig offene Verhältnisse an. Auf lichte Verhältnisse verweisen auch verschiedene NBP-Sippen (Plantago, Artemisia, Thalictrum, Scleranthus annuus, Helianthemum, alles apokrate Sippen. Von Betula gehört zumindest ein Teil zu Betula nana. Wir haben es also mit einer offenen, kräuterreichen und eher steppenartigen Vegetation zu tun und weniger mit Heiden. Ähnliche Vegetationstypen sind im kontinentalen Teil Westgrönlands heute noch zu finden (BÖCHER 1954, 1963). Ein Problem gibt dabei der hohe Anteil der Poaceae auf, der in der Zone 2 sogar bis über $80 \%$ geht. Diese Poaceae könnten natürlich einerseits Bestandteil der Kräutersteppen sein, andererseits ist aber auch an Phragmites zu denken. Und, was uns zumindest wahrscheinlicher erscheint, ohne es beweisen zu können, ist, daß der Gräserpollen aus beiden Quellen stammt. Es scheint, daß der Rückgang des Baumpollens in der Zone 2 wahrscheinlich ein rechnerisches Ergebnis ist und durch die Dominanz der Gräser verursacht wird, in Wirklichkeit aber gar nicht stattgefunden hat. Dafür spricht vor allem auch, daß verschiedene NBP-Typen ebenfalls zurückgehen, aber nicht etwa verschwinden (siehe die Gruppe der Apokraten) und, sobald die Gräser zurückgehen (Zone 3), wieder höhere Werte erreichen.

In der Zone 3 beginnt eine Wiederbewaldung mit einer Sukzession über eine Birkenphase. Die Wiederbewaldung kulminiert im Diagramm in der Zone 4 mit dem Föhrenwald. Föhren sind außer durch Pollen auch durch Spaltöffnungen nachgewiesen worden. Aufgrund des NBP-Spektrums dürfte es sich um einen lockeren, trockenen Föhrenwald gehandelt haben, wahrscheinlich sogar ganz vereinzelt mit Lärchen. Charakteristisch ist, daß mit dem Anstieg und der Dominanz der Föhrenkurve sich auch die Fichtenkurve stabilisiert, allerdings auf einem sehr geringen Niveau. Fichtenpollen wurde über das gesamte Profil hinweg hin und wieder gefunden. Der unterste, maximale Wert beruht aber auf der geringen Pollensumme. Es handelt sich, wie erwähnt, um ein einziges Korn. Die durchgehende Kurve tritt jedoch erst in der Zone 4 in Erscheinung. Da keine Makroreste gefun- den wurden, ist unsicher, ob die Fichte in der Gegend überhaupt vorkam. Die hohen Pollensummen sprechen dafür, daß es sich nicht um umgelagerte Körner handelt. Fernflug kann aber durchaus die zutreffende Interpretation sein.

Bei der Entwicklung vom offenen Grasland zum Föhrenwald fragt es sich, ob sich dahinter ein regional gültiges Prinzip versteckt oder ob es sich nur um eine ganz lokale Erscheinung handelt. Kurz, wurde hier eine generelle, klimagesteuerte Sukzession gefunden oder eine, die nur einen bestimmten Raum wie eine größere Schotter- und Sandfläche betrifft? Die Sedimentationsraten sind nicht bekannt. Aus diesem Grunde kann kein Polleninflux berechnet werden, der das bereits diskutierte Problem mit dem Gräserpollen lösen würde. Die Sedimentationsrate kann in dem Falle auch nicht über ${ }^{14} \mathrm{C}$-Analysen bestimmt werden, denn die Mutungsintervalle wären mit Sicherheit größer als die Zeitdifferenz zwischen der Unter- und der Oberkante des Profils. Das aufgeworfene Problem läßt sich aber mit einem anderen Gedankengang etwas klären. Angenommen, es würde sich hier nur die Vegetationsentwicklung von einem Umkreis von etwa einem Kilometer abzeichnen, so bedeutet dies, daß die angrenzenden Hänge des Odenwaldes und vor allem auch die höher gelegenen Terrassen des Rheins und des Neckars mit Föhren bestockt gewesen sind. Die Optimalphase des Diagrammes belegt ja den Föhrenwald. Dann müßte aber der Anteil des Föhrenpollens in den Zonen 1 und 2 wesentlich höher sein. Das zeigen entsprechende Beispiele aus den Alpen und auch Nordschwedens (KÜTTEL 1979, 1984a). Daher nehmen wir an, daß sich im Diagramm Rösbach eine klimagesteuerte Entwicklung verbirgt. Im Vergleich zur heutigen Vegetation der Gegend muß die Optimalphase des Profils als Interstadial und der davorliegende Abschnitt (die Zonen 1 und 2) als Stadial aufgefaßt werden. Der gesamte Ausschnitt ist eindeutig kontinental getönt. Im Interstadial wird die Temperatur des wärmsten Monates um $10^{\circ} \mathrm{C}$ (Bewaldung) und im Stadial davor deutlich darunter gelegen haben. Das sind fast $10^{\circ} \mathrm{C}$, respektive mehr als $10^{\circ} \mathrm{C}$ weniger als heute.

\subsubsection{Diskussion der Großreste}

Großreste spiegeln vor allem die lokalen Vegetationseinheiten wider. Ein Transport findet nur selten über größere Entfernungen statt. Dies gilt ganz besonders für die meisten Moose. Sie eignen sich deshalb ausgezeichnet zur Rekonstruktion der lokalen Standortsverhältnisse. Bei Früchten und Samen muß man bei der Interpretation schon etwas vorsichtiger sein. Viele Pflanzen bilden z. B. ausgesprochen Flugoder Schwimmfrüchte aus, die durch den Wind, das 
Wasser etc. in andere Vegetationseinheiten verfrachtet werden können.

In der untersten, der Drepanocladus c. f. aduncusZone dürfte die Wasserfläche noch recht neu und offen gewesen sein. Dafür spricht vor allem die namengebende Art, die als Erstbesiedler auf Unterwasser-Sandböden auftritt. Daß die Wasserfläche noch recht jung und wenig von Pflanzen besiedelt war, erkennt man auch am niedrigen Stickstoff- und hohen Siliziumgehalt. Bei der Zone r-2 handelt es sich um ein kalkreiches, offenes Gewässer mit stehendem Wasser. Erst in der zweiten Hälfte treten Menyanthes trifoliata und Sparganium minimum auf, die dann den Characeen das Licht einschränken. S. minimum findet sich nach OBERDORFER (1983) in Wasser von $20-120 \mathrm{~cm}$ Tiefe. Die Pflanze blüht über dem Wasserspiegel und wird vom Wind bestäubt. Da hier Früchte gefunden wurden, ist im mittleren Bereich der Zone mit Wassertiefen nicht über einem Meter zu rechnen. Mit dem Auftreten von Menyanthes wurde das Wasser noch flacher. In der Verlandungszone r-3 klingen die Arten Sparganium minimum und Potamogeton natans aus, um dann in der nächsten Zone fast reinen Moosrasen Platz zu machen. Wasser war zu dieser Zeit nur noch mit geringer Tiefe vorhanden. Zeitweise kann die Lokalität sogar trocken gefallen sein. Scorpidium scorpioides ist charakteristisch für sehr basenreiches Wasser, in dem es auch leicht fluten kann. Calliergon stramineum findet sich als Begleiter in vielen Moosrasen, ohne direkte Hinweise auf Standortsverhältnisse zu liefern.

Wenig läßt sich aus dem Vorkommen von Carex vesicaria ableiten. Sie findet sich vor allem im Verlandungsgürtel von Seen. Ihre Früchte dürften aber vom Wasser recht weit verfrachtet werden, ebenso die von Menyanthes trifoliata.

Recht wenig läßt sich wegen des schlechten Erhaltungszustandes zu den Früchten von Betula sagen, da die Flügel weitgehend fehlten. Aus der Form der Früchte ließ sich aber ein $B$. nana/humilis- und ein $B$. pendula/pubescens-Typ unterscheiden. Wegen der geringen Anzahl der gefundenen Früchte und der Unsicherheit bei der Zuordnung wurde aber im Diagramm darauf verzichtet, sie getrennt darzustellen. Erwähnenswert ist noch das Aussetzen vieler Arten in den Makrorest-Proben 23 und $24 \mathrm{~cm}$, was auch eine Entsprechung in den chemischen Analysen beim Stickstoffgehalt findet. Entsprechend nimmt hier der Asche- und der Siliziumgehalt zu. Im Pollendiagramm setzt etwa in diesem Bereich die EquisetumKurve aus. Es könnte sich um eine kurzzeitige Sandschüttung und damit um eine reine Verdünnung handeln. Die Erklärung des Phänomens bleibt weiteren Untersuchungen vorbehalten.
Auf Grund der Großrestanalysen kann man erkennen, daß es sich beim Profil Rösbach um eine Verlandungsfolge von einem kalkreichen stehenden Gewässer zu einem basenreichen Niedermoor handelt.

\subsubsection{Altersstellung des Profils Rösbach}

Für die Alterseinstufung des Profils auf pollenanalytischem Wege sind folgende Prämissen notwendig:

1) Die Sequenz ist nicht älter als das letzte Interglazial, das heißt nicht älter als Riss/Würm.

2) Die Vegetationsentwicklung, die eindeutig gekappt ist, geht nicht in eine Picea-Phase über.

$\mathrm{Daß}$ die Prämisse 1 zutrifft, geht aus der geologischen Situation hervor. Prämisse 2 ist insofern wahrscheinlich, weil, wenn es sich um einen frühen würmzeitlichen Abschnitt handeln würde, vermehrt Pollen von Picea und thermophiler Gehölze vorhanden sein müßte (vgl. die Diagramme von WoILlaRD 1975; WELTEN 1982; KÜTTEL 1983). Andrerseits kann der interstadiale Abschnitt nicht aus dem Würm-Spätglazial stammen, weil dann sowohl Larix als auch Picea fehlen müßten. Im übrigen wird ein spätglaziales Alter auch durch die Hangendserie widerlegt. Mithin wird das gesamte Rösbach-Profil aus dem Mittelwürm stammen. Diese aus den pollenanalytischen Daten abgeleitete Interpretation wird durch die ${ }^{14} \mathrm{C}$ Daten bestätigt, denn der Torf wurde mit $35640 \pm$ 1500 a BP und Konchylien im Hangenden (s. Abb. 3) mit $31900 \pm 1000$ datiert. Diese Ergebnisse sind mit der pollenanalytischen Interpretation durchaus kohärent. Innerhalb des Mittelwürms ist eine genauere Einstufung aufgrund der pollenanalytischen Daten allein nur schwer möglich. Die ${ }^{14} \mathrm{C}$-Daten hingegen erlauben eine Parallelisation mit dem Hengelo-Interstadial der Niederlande (VOGEL \& ZAGWIJN 1967; VAN DER HAMMEN et al. 1971) welches WOILLARD (1975) mit dem Pile-Interstadial der Grande Pile korreliert. Auch diese Korrelation ist mit den pollenanalytischen Daten konsistent. Für Hengelo wird ein Alter von $39 \mathrm{ka}-37 \mathrm{ka} \mathrm{BP}$ angegeben. Eine Korrelation mit dem Denekamp-Interstadial ist von den ${ }^{14} \mathrm{C}$ Daten her grundsätzlich nicht auszuschließen, doch deuten die lithologischen Verbandsverhältnisse eher auf das Hengelo-Interstadial hin.

\subsubsection{Einzelproben}

\subsubsection{Vegetationsverhältnisse}

Versucht man, auch die Pollenspektren der Einzelproben und kurzen Profilserien in Vegetation umzusetzen, so ergibt sich im Vergleich zum Rösbach-Profil 
ein völlig anderes Bild. Alle Proben lieferten Waldpollenspektren, wobei die Nr. 6 aufgrund der Kombination von Pinus und Artemisia etwas aus dem Rahmen fällt. Problematisch ist für die weitere Deutung aber, daß Pollenspektren immer integrierende Abbilder der Vegetationsverhältnisse eines ganzen Landschaftsraumes ergeben. Das heißt, es kann nicht unterschieden werden, ob es sich hier um gut durchmischte Laubmischwälder mit hohem Koniferenanteil gehandelt hat, oder, was wir für wahrscheinlicher halten, eine reiche standortliche Differenzierung vorhanden war. Beides deutet aber auf klimatische Verhältnisse hin, die den heutigen nicht entsprechen. Es wird insgesamt kühler gewesen sein, aber bedeutend wärmer im Vergleich zu den Verhältnissen, die das Profil Rösbach auch im Optimum anzeigt. Im Vergleich zu heute dürften endinterglaziale oder warme Interstadialbedingungen zutreffen.

\subsubsection{Zeitliche Einstufung}

Für die zeitliche Einstufung der Einzelproben sind wenig klare Hinweise vorhanden. Aufgrund der weitgehend fehlenden Buche und der Häufigkeit der Fichte ist Holozän eindeutig auszuschließen. Mittelwürm ist ebenfalls auszuschließen. Das zeigen die Diagramme der Grande Pile (Woillard 1975) und des Schweizer Mittellandes (WeLTEN 1982; KÜTTEL 1983). Daraus ergibt sich eine Zeitstellung vor dem Mittelwürm. Die zwei folgenden Hypothesen kommen in Betracht:

1) Die siltigen Gyttjen sind allesamt oder teilweise älter als Riß/Würm. Möglich wären warme Interstadiale innerhalb der Rißeiszeit. Eine Korrelation mit noch älteren Ablagerungen, wie etwa denen von Steinbach (SCHEDLER 1981), benötigte etliche zusätzliche Hilfshypothesen und kommt kaum in Betracht.

2) Die Proben stammen aus dem Spät-Riß/Würm und/oder aus dem Frühwürm (hier natürlich aus den Interstadialen).

Die erste Hypothese ist dann auszuschließen, wenn die zeitliche Einstufung der von LÖSCHER et al. (1983: 69) erwähnten Funde zutrifft. Wir halten dies für gerechtfertigt und somit die zweite Hypothese für wahrscheinlicher. Eine feinere Einstufung ist aber schwerlich möglich, ausgenommen, daß die Proben im Vergleich zu den Diagrammen der Grande Pile (WOILLARD 1975) stets aus den Abkühlphasen stammen müßten. Möglich sind Eem, St. Germain I und St. Germain II. Eine letzte Variante, für die es jedoch keine Hinweise gibt, ist, daß die Einzelspektren aus vegetationsgeschichtlichen Abschnitten stammen, die in den Profilen der Grande Pile nicht enthalten sind (Hiaten) oder ungenügend entwickelt sind. Grund- sätzlich ist nicht auszuschließen, daß Brörup und Odderade in den Profilen der Grande Pile ausnahmslos durch Hiaten repräsentiert sind. Nur, es fehlen klare Befunde, nicht einmal Hinweise sind vorhanden.

Abschließend kann festgehalten werden, daß ein föhrenreiches Mittelwürm-Interstadial nachgewiesen wurde. Die Einzelproben hingegen können sowohl aus dem Riß/Würm-Interglazial und dem Frühwürm stammen. Mit den bisher vorliegenden Pollenanalysen kann die Einstufung der eichenführenden Schicht (vgl. Abb. 2) als ganzes ins Eem, resp. Riß/Würm, weder gestützt noch widerlegt werden.

\section{Dank}

Wir danken Herrn dipl. Agraring A. Tsobanidis, Stuttgart, für die Aufbereitung der Proben für die Pollenanalysen und Herrn Dipl. Bibl. H. ADAM für die Reinschrift des Manuskripts.

\section{Schriftenverzeichnis}

Aalto, M. (1970): Potamogetonaceae fruits. I. Recent and subfossil endocarps of the fennoscandian species. Acta Bot. Fenn., 88: 1-85; Helsinki.

ALLen, S. E. (Edit.) (1974): Chemical analysis of ecological materials. - 565 S.; Oxford (Blackwell).

ARMBRuster, S., JoACHim, H., LAMprecht, K. \& Villinger, E. (1977): Grenzen der Grundwassernutzung im RheinNeckar-Raum (Süd.-Württ.). —Z. dt. geol. Ges., 128: 263-296; Hannover.

BAAS, J. (1938): Zur Geschichte der Pflanzenwelt und der Haustiere im unteren Maintal. - Abh. Senckenberg, Natf. Ges., 440: 1-36; Frankfurt/Main.

BARTZ, J. (1959): Zur Gliederung des Pleistozäns im Oberrheingebiet. - Z. dt. geol. Ges., 111: 653-661; Hannover.

BARTZ, J. (1974): Die Mächtigkeit des Quartärs im Oberrheingraben. - In: IluIEs, J. H. \& FucHs, K.: Approaches to Taphrogenesis, S. 78-87; Stuttgart (Schweizerbart).

BARTZ, J. mit Beiträgen von G. v. d. BRelie \& H. Maus (1976): Quartär und Jungquartär im Raum Rastatt. Jb. geol. L. A. Bad.-Württ., 18: 121-178; Freiburg i. Br.

BARTZ, J. (1982): Quartär und Jungquartär II im Oberrheingraben im Großraum Karlsruhe. - Geol. Jb., 63: 237 S.; Hannover.

BERGGREN, G. (1969): Atlas of seed and small fruits of northwest-european plant species with morphological descriptions. Part 2: Cyperaceae. - 68 S.; Stockholm. 
BerTSCH, K. (1941): Früchte und Samen. Ein Bestimmungsbuch zur Pflanzenkunde der vorgeschichtlichen Zeit. Handbücher d. praktischen Vorgeschichtsforschung, 1, 247 S.; Stuttgart.

BÖCHER, T. W. (1954): Oceanic and continental vegetational complexes in Southwest Greenland. - Medd. o. Gronland, 147.2: 1-63; Kopenhagen.

BÖCHER, T. W. (1963): Phytogeography of middle west Greenland. Medd. o. Gr $\phi$ nland, 148.3: 1-289; Kopenhagen.

FIRBAS, F. (1934): Zur spät- und nacheiszeitlichen Vegetationsgeschichte der Rheinpfalz. - Beih. Bot. Cbl., 52: 119-159; Jena.

FrENZEL, B. (1964): Zur Pollenanalyse von Lößen. Untersuchungen der Lößprofile von Oberfellabrunn und Stillfried (Niederösterreich). - Eiszeitalter und Gegenwart, 15: 5-39; Hannover.

Grosse-BrauckmanN, G. (1972): Über pflanzliche Makrofossilien mitteleuropäischer Torfe. I: Gewebereste krautiger Pflanzen und ihre Merkmale. - Telma, 2, 19-55; Hannover.

HAMMEN, T. VAN DER, WijMSTRA, T. A. \& ZAGWijn, W. H. (1971): The floral record of the Late Cenozoic of Europe. - In: TureKian, K. K. (ed.): Late Cenozoic Glacial Ages, S. 391-424; New Haven (Yale University Press).

HÖlzer, A. \& SCHLOSs, S. (1981): Paläoökologische Studien an der Hornisgrinde (Nordschwarzwald) auf der Grundlage von chemischer Analyse, Pollen- und Großrestuntersuchung. - Telma, 11: 17-30; Hannover.

KAC, N. JA., KaC, S. V. \& KiPIANI, M. G. (1965): Atlas i opredelitel plodov i semjan, vstrečajuscichsja v četverticnych otloženijach SSSR. - 365 S.; Moskau.

KOENIGSWALD, W. v. \& LÖSCHER, M. (1982): Jungpleistozäne Hippopotamus-Funde aus der Oberrheinebene und ihre biogeographische Bedeutung. - N. Jb. Geol. Paläont. Abh., 163: 331-348; Stuttgart.

KUTTEL, M. (1979): Pollenanalytische Untersuchungen zur Vegetationsgeschichte und zum Gletscherrückzug in den westlichen Schweizer Alpen. - Ber. Schweiz. Bot. Ges., 89: 9-62; Teufen.

KƯTTEL, M. (1983): Züge der jungpleistozänen Vegetations- und Landschaftsgeschichte der Zentralschweiz. Manuskript, $215 \mathrm{~S}$.

KÜTtel, M. (1984a): Vuolep Allakasjaure - eine pollenanalytische Studie zur Vegetationsgeschichte der Tundra in Nordschweden. - In LANG, G. (ed.): Festschrift Max Welten, Diss. Bot., 72: 191-212; Vaduz.

KÜTTEL, M. (1984b): Veränderung von Diversität und Evenness der Tundra aufgezeichnet im Pollendiagramm des Vuolep Allakasjaure. - Botanica Helvetica, 94: 279-283; Basel.

LessmanN, U. (1983): Pollenanalyse an Böden im nördlichen Oberrheintal unter besonderer Berücksichtigung der Steppenböden. - Diss. Uni Bonn, 108 S.
LÖSCHER, M. (1981): Die stratigraphische Gliederung des Jungpleistozäns im Neckarschwemmfächer bei Heidelberg. - Aufschluß, 32: 191-199; Heidelberg.

LÖscher, M., Becker, B., Bruns, M., Hieronymus, U., MÄUSBACHER, R., MÜNNICH, M., MUUNZING, K. \& SCHEDLER, J. (1980): Neue Ergebnisse über das Jungquartär im Neckarschwemmfächer bei Heidelberg. - Eiszeitalter und Gegenwart, 30: 89-100; Hannover.

LÖSCHER, M., CORDES, U. \& SCHLOSS, S. (1983): Holozäne und jungpleistozäne Sedimente im Oberrheingraben bei Heidelberg. - Geol. Jb., A 71: 61-72; Hannover.

MÚller, T., Oberdorfer, E. \& PhilipPI, G. (1974): Die potentielle Vegetation von Baden-Württemberg. - Beihefte zu den Veröffentlichungen der Landesstelle für Naturschutz und Landschaftspflege Baden-Württemberg, 6: 45 S.; Ludwigsburg.

NiLsson, Ö. \& HJelmQvist, H. (1967): Studies on the nutlet structure of south scandinavian species of Carex. - Bot. Notiser, 120: 460—485; Lund.

NyноLм, E. (1954-1969): Illustrated moss flora of Fennoscandia. 2: Musci. - 799 S.; Lund.

Oberdorfer, E. (1934): Zur Geschichte der Sümpfe und Wälder zwischen Mannheim und Karlsruhe. - Ver. f. Naturkunde Mannheim (Festschrift zur 100-Jahr-Feier), 100/101; 99-124; Mannheim.

OBERDORFER, E. $\left(1983^{5}\right)$ : Pflanzensoziologische Exkursionsflora. - 1051 S.; Stuttgart (Ulmer).

RothSCHILD, S. (1936): Zur Geschichte der Moore und Wälder der oberrheinischen Tiefebene. - Beih. Bot. Cbl., 54 Abt. B: 140-184; Jena.

SCHEDLER, J. (1981): Vegetationsgeschichtliche Untersuchungen an altpleistozänen Ablagerungen in Südwestdeutschland. - Diss. Bot. 58: 158 S.; Vaduz.

SCHNEIDER, E. \& SCHNEIDER, L. (1975): Synsedimentäre Bruchtektonik des Oberrheingrabens zwischen Speyer, Worms, Hardt und Odenwald. - Münster. Forsch. Geol. Paläont., 36: 81-126; Münster/Westf.

STARK, P. (1926): Ein altes Moorprofil im Oberrheintal bei Mannheim. Ber. Natf. Ges. Freiburg, 44: 373-376; Freiburg.

Vogel, J. C. \& ZaGwiJn, W. H. (1967): Groningen Radiocarbon Dates VI. - Radiocarbon, 9: 63-106; New Haven.

Wetten, M. (1982): Pollenanalytische Untersuchungen im jüngeren Quartär des nördlichen Alpenvorlandes der Schweiz. - Beitr. geol. Karte Schweiz N.F., 156: 174 S.; Bern.

WOILLARD, G. (1975): Recherches palynologiques sur le pleistocène dans l'est de la Belgique et dans les Vosges Lorraines. - Acta Geogr. Lov., 14: 1-118; Louvain.

Manuskript eingegangen am 4. 6. 1985,

Nachträge Dezember 1985 